\title{
LIGHT OPEN AND OPEN MAPPINGS ON MANIFOLDS. II
}

\author{
BY
}

JOHN J. WALSH

\begin{abstract}
Sufficient conditions are given for the existence of light open mappings between p.l. manifolds. In addition, it is shown that a mapping $f$ from a p.l. manifold $M^{m}, m \geqslant 3$, to a polyhedron $Q$ is homotopic to an open mapping of $M$ onto $Q$ iff the index of $f_{*}\left(\pi_{1}(M)\right)$ in $\pi_{1}(Q)$ is finite. Finally, it is shown that an open mapping of $M^{m}$ onto a p.1. manifold $N^{n}, n>m>3$, can be approximated by a light open mapping of $M$ onto $N$.
\end{abstract}

In [19], D. Wilson constructs examples of light open mappings (with each point inverse a Cantor set) from any 3 manifold onto any $n$ cell $(n \geqslant 3)$ and he constructs examples of monotone open mappings of any p.1. manifold $M^{m}$ $(m \geqslant 3)$ onto any $n$ cell (these results answered questions raised by Eilenberg in [3]). In the first paper in this series [24], the author gave a complete analysis of the existence of monotone and monotone open mappings from manifolds onto polyhedra. In this paper, we give a complete analysis of the existence of open mappings from manifolds onto polyhedra (using results from [24] and from the theory of covering spaces); however, the principal content of this paper is the technique developed in $\S 5$ for constructing light open mappings between manifolds (with each point inverse a Cantor set). The techniques used in this paper are inspired by those of D. Wilson in [19] and [18]; indeed, the many similarities are apparent.

The "key" result which enables us to remove the assumption (of Wilson in [19]) that the domain manifold have dimension three is contained in the appendix (it is necessary to study $\S 5$ in order to understand the relevance of the appendix). The philosophy behind removing the assumption that the image is a cell is exactly the same as in [24] (however, we must assume the image is a manifold). In addition, the technical difficulties encountered in $\S 5$ are numerous.

In order to read this paper, it will be necessary to have a copy of the first part [24]. Indeed, we will need to refer to [24] so often that we have numbered the sections of this paper beginning with 4; any reference to $\S 1,2$, or 3 will be to that section of [24] (eg., (3.7.1) refers to $\S 3$ of [24]). The notation used here is exactly as in [24], hence we shall not reproduce it.

Received by the editors August 26, 1974 and, in revised form, March 21, 1975. AMS (MOS) subject classifications (1970). Primary 54C10; Secondary 57C99.

Key words and phrases. Light open mapping, open mapping, p.l. manifold, polyhedron. 
4. In this section, we develop an "up to homotopy" monotone open-light open factorization which leads to a proof of the following theorem.

(4.0) THEOREM. A mapping from a compact, connected p.l. manifold $M^{m}, m \geqslant 3$, to a compact, connected polyhedron $Q$ is homotopic to an open mapping of $M$ onto $Q$ if and only if the index of $f_{*}\left(\pi_{1}(M)\right)$ in $\pi_{1}(Q)$ is finite.

The "only if" half of this result is due to Smale [22]; we present in Proposition (4.1) a modified version of Smale's result which we prove using covering space theory. The reader is referrred to [23] for results on covering spaces used below. Recall that a mapping is proper provided the inverse image of each compact set is compact.

(4.1) Proposition. Let $X$ and $Y$ be connected metric spaces with $Y$ semilocally simply connected and let $f:\left(X, x_{0}\right) \rightarrow\left(Y, y_{0}\right)$ be a proper, open mapping which is also onto. Then the index of $f_{*}\left(\pi_{1}\left(X, x_{0}\right)\right)$ in $\pi_{1}\left(Y, y_{0}\right)$ is finite.

Proof. Let $p:\left(\tilde{Y}, \tilde{y}_{0}\right) \rightarrow\left(Y, y_{0}\right)$ be a covering projection with $p_{*}\left(\pi_{1}\left(\widetilde{Y}, \tilde{y}_{0}\right)\right)=f_{*}\left(\pi_{1}\left(X, x_{0}\right)\right)$; recall that the index of $f_{*}\left(\pi_{1}\left(X, x_{0}\right)\right)$ in $\pi_{1}\left(Y, y_{0}\right)$ is equal to the cardinality of $p^{-1}\left(y_{0}\right)$. Let $\tilde{f}:\left(X, x_{0}\right) \rightarrow\left(\widetilde{Y}, \tilde{y}_{0}\right)$ be a lifting of $f$; that is, $p \circ \widetilde{f}=f$. It follows that $\tilde{f}$ is proper and open and, hence, onto (recall that $\widetilde{Y}$ is connected). Since $\tilde{f}^{-1}\left(p^{-1}\left(y_{0}\right)\right)=f^{-1}\left(y_{0}\right)$ is compact, it follows that $p^{-1}\left(y_{0}\right)$ is compact and, hence, finite.

Proof OF TheOREM (4.0). The "if" half is proved as follows. Let $p$ : $\left(\widetilde{Q}, \widetilde{q}_{0}\right) \rightarrow\left(Q, q_{0}\right)$ be a covering projection with $p_{*}\left(\pi_{1}\left(\widetilde{Q}, \widetilde{q}_{0}\right)\right)=f_{*}\left(\pi_{1}\left(M, x_{0}\right)\right)$. Since the index of $f_{*}\left(\pi_{1}\left(M, x_{0}\right)\right)$ in $\pi_{1}\left(Q, q_{0}\right)$ is finite, we have that $p^{-1}\left(q_{0}\right)$ is finite; hence $\widetilde{Q}$ is a compact, connected polyhedron. Let $\widetilde{f}:\left(M, x_{0}\right) \rightarrow$ $\left(\widetilde{Q}, \tilde{y}_{0}\right)$ be a lifting of $f$. It follows that $\widetilde{f}_{*}: \pi_{1}\left(M, x_{0}\right) \rightarrow \pi_{1}\left(\widetilde{Q}, \widetilde{q}_{0}\right)$ is onto. Corollary (3.7.2) in [24] implies that $\tilde{f}$ is homotopic to a monotone open mapping $\tilde{g}$ from $M$ onto $\widetilde{Q}$ (we do not claim that $\tilde{g}$ preserves base points). Let $g=p \circ \tilde{g}$; then $g$ is homotopic to $f$ and $g$ is an open mapping of $M$ onto $Q$. As promised earlier, we may view $p \circ \tilde{g}$ as a monotone open-light open factorization of $f$ (up to homotopy).

5. This section closely parallels $\S 3$ in [24] ; Proposition (5.1) below contains the main technical tool for constructing light open mappings (as Proposition (3.1) did for constructing open mappings). Theorem (5.0) follows from Proposition (5.1) (and its proof) and Proposition 3 in [18]. Observe that the only difference between the hypothesis of the following theorem and that of Theorem (3.0) is that we assume that the range is a p.l. manifold of sufficient dimension, not simply a polyhedron. 
(5.0) THEOREM. Let $M^{m}, N^{n}$ be compact, connected p.l. manifolds $(n \geqslant m \geqslant 3)$ with triangulations $K$ and $L$, respectively. Let $P$ be a collection of nonempty subsets of $M$ with pairwise disjoint interiors, with each $p \in P$ anion of elements of $t(K)$, and with $P^{*}=M$. Let $T$ be a one to one function from $P$ onto $t(L)$ satisfying:

(5.0.1) $T\left(p_{1}\right) \cap \ldots \cap T\left(p_{q}\right) \neq \varnothing$ whenever $p_{1} \cap \ldots \cap p_{q} \neq \varnothing$.

(5.0.2) Each component of $T^{-1}\left(\sigma_{1}\right)$ meets $T^{-1}\left(\sigma_{2}\right)$ whenever $\sigma_{1} \cap \sigma_{2}$ $\neq \varnothing, \sigma_{1}, \sigma_{2} \in t(L)$.

Then there is a light open mapping $f$ from $M$ onto $N$ with $f^{-1}(y)$ homeomorphic to a Cantor set for each $y \in N$ and with $f(x) \in \mathrm{st}^{2}(T(p), L)^{*}$ for $x \in$ $p \in P$.

It is best to view Proposition (5.1) as a (nontrivial) modification of Proposition (3.1). To this end, we will indicate how to modify the first part of the proof of (3.1); that is, up to but not including (3.5). At this point the two proofs differ radically and we will continue in detail with the remainder of the proof of (5.1).

(5.1) Proposition. Assume the hypothesis of Theorem (5.0); then there are two sequences of finite collections of polyhedra $\left\{J_{n}\right\}_{n=1}^{\infty}$ and $\left\{K_{n}\right\}_{n=1}^{\infty}$ satisfying: (5.1.1),(5.1.2), . . , (5.1.7) are exactly the same as (3.1.1), (3.1.2), $\ldots,(3.1 .7)$.

(5.1.8) If $j_{n}^{1} \cap \ldots \cap j_{n}^{r} \neq \varnothing$, then the diameter of each component of $R_{n}\left(j_{n}^{1}\right) \cup \ldots \cup R_{n}\left(j_{n}^{r}\right)$ is less than $28 / 2^{n-1}$.

Proof of (5.1). We shall use $(5.1 .)^{n}$ to indicate condition $(5.1 . \cdot)$ for the $n$th stage. The construction for $n=1$ is done as in (3.1); we now proceed from the $n$th stage to the $(n+1)$ st stage.

(5.2) Choose $H$ and $\epsilon^{\prime}$ as in (3.2) with the additional requirement that $\mathrm{st}^{4}(a, t(H))^{*} \cap \mathrm{st}^{4}\left(a^{\prime}, t(H)\right)^{*}=\varnothing$ for $a \in A_{j_{n}}, a^{\prime} \in A_{j_{n}^{\prime}}$ and $a \neq a^{\prime}$. We are now going to determine $l_{n+1}$ and $L_{n+1}$; however, in doing so, we will also be setting up a considerable amount of machinery to be used later. The reader is advised to develop a schematic picture while going through the next paragraph; see Figure 1.

Let $Q$ be an integer such that $\mathrm{ST}^{Q}\left(A_{j_{n}}^{*}, t\left(H \mid j_{n}\right)\right)^{*}=R_{n}\left(j_{n}\right)$ for each $j_{n} \in$ $J_{n}$. Choose $l_{n+1}$ as follows. Recall that $\epsilon^{\prime}$ is chosen as in (3.2); let $Q^{\prime}=Q+$ $3 m^{2}+5$ and let $l>l_{n}$ be such that the diameter of each st $Q^{\prime+1}\left(\sigma, \beta^{l} L\right)^{*}$ is less than $\epsilon^{\prime}$ for each $\sigma \in \beta^{l} L$ and such that st $Q^{\prime}\left(j_{n}, \beta^{l} L\right)^{*} \subseteq \operatorname{st}\left(j_{n}, \beta^{l_{n}+1} L\right)^{*}$; this last condition will guarantee the conclusion in (5.0) that $f(x) \in \mathrm{st}^{2}(T(p), t(L))^{*}$. Let $3 \eta=$ minimum of $\left\{\right.$ distance of $N-\operatorname{st}^{i}\left(\sigma, \beta^{l} L\right)^{*}$ to $\mathrm{st}^{i-2}\left(\sigma, \beta^{l} L\right)^{*} \mid \sigma \in$ $\beta^{l} n L$ and $2 \leqslant i \leqslant Q^{\prime}$ \}. Let $l^{\prime}>l+4$ be an integer such that the diameter 


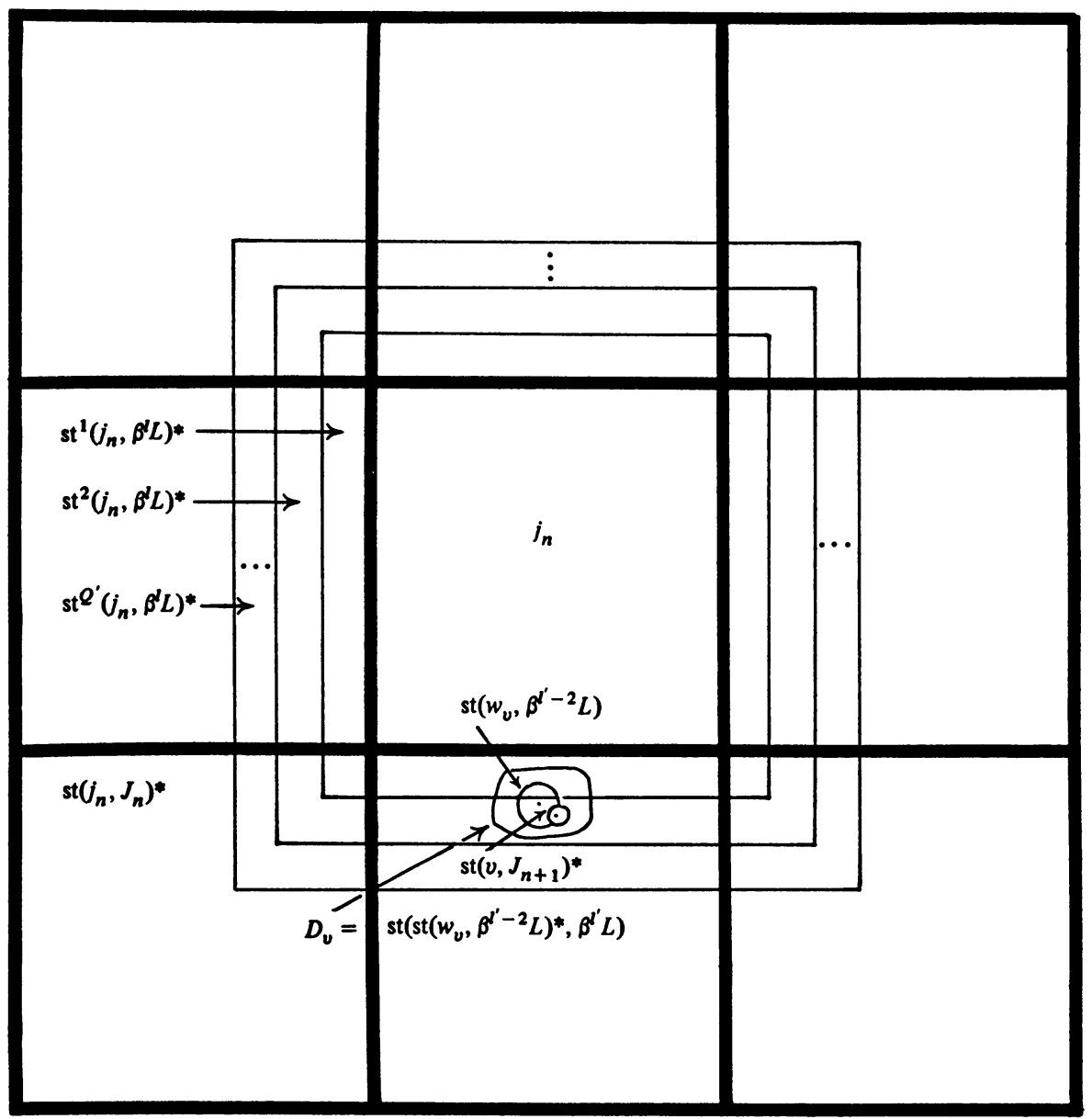

FIGURE 1

of $\operatorname{st}\left(\operatorname{st}\left(w, \beta^{l^{\prime}-2} L\right)^{*}, \beta^{l^{\prime}} L\right)^{*}$ is less than $\eta$ for each vertex $w \in \beta^{l^{\prime}-3} L$. Let $l_{n+1}>l^{\prime}$ be an integer such that if $v$ is a vertex of $\beta^{l} n+1 L$ and $v \in$ $\operatorname{st}\left(w, \beta^{l^{\prime}-2} L\right)^{*}$ for some vertex $w \in \beta^{l^{\prime}-3} L$, then $\operatorname{st}\left(v, \beta^{l n+1} L\right)^{*} \subseteq$ $\operatorname{int}\left(\operatorname{st}\left(\operatorname{st}\left(w, \beta^{l^{\prime}-2} L\right)^{*}, \beta^{l^{\prime}} L\right)^{*}\right)$. Finally, let $L_{n+1}=Q^{\prime} 2^{l_{n+1}-l}$; the reader should check that st ${ }^{L+1}\left(j_{n}, J_{n+1}\right)^{*}=\mathrm{st}^{\prime}\left(j_{n}, t\left(\beta^{l} L\right)\right)^{*}$ and that if $j_{n+1} \in J_{n+1}=$ $\beta^{l_{n+1}} L$ and $\sigma \in t\left(\beta^{l} L\right)$ with $j_{n+1} \subseteq \sigma$, then st ${ }^{L}{ }^{n+1}\left(j_{n+1}, J_{n+1}\right)^{*} \subseteq$ st $^{\prime}\left(\sigma, t\left(\beta^{l} L\right)\right)^{*}$. In particular, the diameter of $\operatorname{st}^{L_{n+1}}\left(j_{n+1}, J_{n+1}\right)^{*}$ is less than $\epsilon^{\prime}$ and, therefore, condition (5.1.2) $)^{n+1}$ holds.

(5.3) Read here exactly the rule stated in (3.3). As in (3.3), this basic rule guarantees that $(5.1 .4)^{n+1}$ will hold and this rule together with (5.1.7) ${ }^{n}$ (and the above choice of $L_{n+1}$ ) guarantees that $(5.1 .5)^{n+1}$ and (5.1.6) will hold. One fact deducible from the choices of $Q, Q^{\prime}$ and $L_{n+1}$ is that if 
$j_{n+1} \subseteq \mathrm{st}^{3 m^{2}}\left(j_{n}, t\left(\beta^{l} L\right)\right)^{*}$, then $R_{n+1}\left(j_{n+1}\right)$ is to meet every member $h \in t(H)$ in $R_{n}\left(j_{n}\right)$.

(5.4) For each vertex $v$ of $J_{n+1}$ (more precisely, $v$ is a vertex of $\beta^{l_{n+1}} L$ ), let $S(v)=\left\{\mathrm{bd}\left(\operatorname{st}\left(w, \beta^{2} H\right)^{*}\right) \mid w \text { : vertex of } \beta^{1} H\right\}^{*}$; each $S(v)$ is a subcomplex of $\beta^{2} H$. General position the $S(v)$ 's with respect to each other and with respect to $\operatorname{cl}\left(H^{m-1} \cap(M-\partial M)\right)$ so that the part of each $S(v)$ in $\partial M$ (resp. $M-\partial M$ ) remains in $\partial M$ (resp. $M-\partial M$ ). Let $S=\bigcup S(v)$ and let $B_{1}$ be a subdivision of $H$ with each $S(v)$ a subcomplex of $B_{1}$.

Let $U=\operatorname{st}\left(S, \beta^{2} B_{1}\right)^{*}$, let $K_{n}^{U}=\left\{k_{n} \cap \operatorname{cl}(M-U) \mid k_{n} \in K_{n}\right\}$, and let $R_{n}^{U}\left(j_{n}\right)=R_{n}\left(j_{n}\right) \cap \operatorname{cl}(M-U) \in K_{n}^{U}$.

(5.5) Most likely, the collection of components of the sets in $K_{n}^{U}$ will not be simple (a fact we will have to live with). By using $l_{n+1}-l_{n}$ applications of the construction in (1.3) (begin with the triple $\left(R_{n}^{U}\right)^{-1}: K_{n}^{U} \rightarrow J_{n}$ which satisfies (1.1.1), but omit the step where the collection of components is made simple), construct a triple $T_{U}: P_{U} \rightarrow J_{n+1}$ satisfying:

(5.5.1) $P_{U}^{*}=\left(K_{n}^{U}\right)^{*}$ and $\operatorname{cl}(\operatorname{int}(p))=p$ for each $p \in P_{U}$; the collection of components of the sets in $P_{U}$ may not be simple.

(5.5.2) If $j_{n+1} \subseteq j_{n}$, then $T_{U}^{-1}\left(j_{n+1}\right) \subseteq R_{n}^{U}\left(j_{n}\right)$.

(5.5.3) $T_{U}\left(p_{1}\right) \cap \ldots \cap T_{U}\left(p_{q}\right) \neq \varnothing$ whenever $p_{1} \cap \ldots \cap p_{q} \neq \varnothing$.

(5.5.4) If $C, C^{\prime}$ are components of $\mathrm{cl}(h-U), \mathrm{cl}\left(h^{\prime}-U\right), h \subseteq R_{n}^{U}\left(j_{n}\right)$ and $h^{\prime} \subseteq R_{n}^{U}\left(j_{n}^{\prime}\right)$, and $C \cap C^{\prime}$ contains an $m-1$ cell, then for each pair $j_{n+1}$ $\subseteq j_{n}, j_{n+1}^{\prime} \subseteq j_{n}^{\prime}$ with $j_{n+1} \cap j_{n+1}^{\prime} \neq \varnothing, \operatorname{int}\left(T_{U}^{-1}\left(j_{n+1}\right) \cup T_{U}^{-1}\left(j_{n+1}^{\prime}\right)\right) \cap$ $\operatorname{int}\left(C \cup C^{\prime}\right)$ is connected (possibly, $C=C^{\prime}, j_{n}=j_{n}^{\prime}$, or $j_{n+1}=j_{n+1}^{\prime}$ ).

By running additional tubes during each application of (1.3), we can assume in addition:

(5.5.5) If $C$ is a component of $\mathrm{cl}(h-U), h \subseteq R_{n}\left(j_{n}\right)$, and $\sigma \subseteq C \cap U$, $\sigma$ an $m-1$ simplex of $\beta^{2} B_{1}$, then $T_{U}^{-1}\left(j_{n+1}\right) \cap \sigma$ contains an $m-1$ cell for each $j_{n+1} \subseteq j_{n}$.

(5.6) We are now going to alter the triple $T_{U}: P_{U} \rightarrow J_{n+1}^{*}$ so that $T_{U}^{-1}\left(j_{n+1}\right)$ meets each set in $t(H)$ which $R_{n+1}\left(j_{n+1}\right)$ is supposed to meet (see (5.3)).

Let $C$ be a component of $\operatorname{cl}(h-U), h \subseteq R_{n}\left(j_{n}\right)$ and $h \in t(H)$; let $r$ be an integer such that $R_{n+1}\left(j_{n+1}\right)$ is to meet $h$ if and only if $j_{n+1} \in \operatorname{st}^{r}\left(j_{n}, J_{n+1}\right)$. For each $j_{n+1} \in \operatorname{st}^{1}\left(j_{n}, J_{n+1}\right)-\operatorname{st}^{0}\left(j_{n}, J_{n+1}\right)$ alter $T_{U}^{-1}\left(j_{n+1}\right)$ by adding to it an $m$ cell in $\operatorname{int}\left(T_{U}^{-1}\left(j_{n+1}^{\prime}\right)\right) \cap \operatorname{int}(C)$ where $j_{n+1} \cap j_{n+1}^{\prime} \neq \varnothing$ and $j_{n+1}^{\prime} \in$ st $^{0}\left(j_{n}, J_{n+1}\right)$; remove the interior of the $m$ cell from $T_{U}^{-1}\left(j_{n+1}^{\prime}\right)$. Inductively, for $i=2, \ldots, r$, if $j_{n+1} \in \mathrm{st}^{i}\left(j_{n}, J_{n+1}\right)-\mathrm{st}^{i-1}\left(j_{n}, J_{n+1}\right)$, then add to $T_{U}^{-1}\left(j_{n+1}\right)$ an $m$ cell in $\operatorname{int}\left(T_{U}^{-1}\left(j_{n+1}^{\prime}\right)\right) \cap \operatorname{int}(C)$ where $j_{n+1} \cap j_{n+1}^{\prime} \neq \varnothing$ and $j_{n+1}^{\prime} \in \mathrm{st}^{i-1}\left(j_{n}, J_{n+1}\right)$; remove the interior of the $m$ cell from $T_{U}^{-1}\left(j_{n+1}^{\prime}\right)$. 
Let $P_{U}^{C}=\left\{\mathrm{cl}\left(T_{U}^{-1}\left(j_{n+1}\right) \cap \operatorname{int}(C)\right) \mid j_{n+1} \in \operatorname{st}^{r}\left(j_{n}, J_{n+1}\right)\right\}$ and let $T_{U}^{C}\left(\mathrm{cl}\left(T_{U}^{-1}\left(j_{n+1}\right) \cap \operatorname{int}(C)\right)\right)=j_{n+1}$ for $j_{n+1} \in \operatorname{st}^{r}\left(j_{n}, J_{n+1}\right)$. Apply the techniques in (2.5) - (2.8) to the triple $T_{U}^{C}: P_{U}^{C} \rightarrow \operatorname{st}^{r}\left(j_{n}, J_{n+1}\right)$, using the fact that $\operatorname{st}^{r}\left(j_{n}, J_{n+1}\right)^{*}$ is simple connected, to alter the sets $T_{U}^{-1}\left(j_{n+1}\right), j_{n+1} \in$ $\operatorname{st}^{r}\left(j_{n}, J_{n+1}\right)$ so that:

(5.6.1) If $j_{n+1}, j_{n+1}^{\prime} \in \operatorname{st}^{r}\left(j_{n}, J_{n+1}\right)$ with $j_{n+1} \cap j_{n+1}^{\prime} \neq \varnothing$, then $\operatorname{int}\left(T_{U}^{-1}\left(j_{n+1}\right) \cup T_{U}^{-1}\left(j_{n+1}^{\prime}\right)\right) \cap \operatorname{int}(C)$ is connected.

(5.7) After the alterations of (5.6) have been made for each $h \in t(H)$ and each component $C$ of cl $(h-U)$, the altered triple $T_{U}: P_{U} \rightarrow J_{n+1}$ will still satisfy (5.5.1), (5.5.3), (5.5.4), and (5.6.1); be warned that the superscript " $r$ " in condition (5.6.1) depends on the set $h$ for which we have $C$ a component of $\mathrm{cl}(h-U)$. In addition, $T_{U}^{-1}\left(j_{n+1}\right)$ meets every member of $t(H)$ which $R_{n+1}\left(j_{n+1}\right)$ is to meet and $T_{U}^{-1}\left(j_{n+1}\right) \cap H^{m-1} \subseteq R_{n}\left(j_{n}\right)$ where $j_{n+1} \subseteq j_{n}$.

Assessing what we have done so far, we observe that the triple $T_{U}: P_{U} \rightarrow$ $J_{n+1}$ satisfies all the conditions (5.1. $)^{n+1}$ (with $T_{U}^{-1}$ and $P_{U}$ in place of $R_{n+1}$ and $K_{n+1}$ ) except for (5.1.1) $)^{n+1}$ and (5.1.7) $)^{n+1}$. The two major problems we face are that $P_{U}^{*} \neq M$ and that each component of $T_{U}^{-1}\left(j_{n+1}\right)$ may not meet $T_{U}^{-1}\left(j_{n+1}^{\prime}\right)$ even though $j_{n+1}^{\prime} \cap j_{n+1} \neq \varnothing$. $\S \S(5.8)-(5.11)$ deal with the latter problem and (5.12) the former.

Before proceeding, let us discuss the role of the $S(v)$ 's. Ideally, we would like $R_{n+1}\left(j_{n+1}\right) \cap S(v)=\varnothing$ for each $v \in j_{n+1}$ as this would immediately give us $(5.1 .8)^{n+1}$. We will not have this ideal situation, but we will "control" this intersection so that we can still deduce (5.1.8) $)^{n+1}$.

(5.8) Let $v$ be a vertex of $J_{n+1}$ and let $h \in t(H)$ where $h \subseteq R_{n}\left(j_{n}\right)$. For each pair $D, D^{\prime}$ of components of $h-S$ with $\mathrm{cl}(D) \cap \mathrm{cl}\left(D^{\prime}\right)$ containing an $m-1$ cell in $S(v)$ make the following alterations. (Let $C, C^{\prime}$ be the unique components of $h-U$ with $C \subseteq D, C^{\prime} \subseteq D^{\prime}$.) For each $j_{n+1} \subseteq j_{n}$ with $j_{n+1} \cap N_{\eta}(v)$ $=\varnothing$ (recall that $\eta$ was defined in (5.2)), use condition (5.5.5) to connect $T_{U}^{-1}\left(j_{n+1}\right) \cap \operatorname{cl}(C)$ to $T_{U}^{-1}\left(j_{n+1}\right) \cap \operatorname{cl}\left(C^{\prime}\right)$ with a tube in $T_{U}^{-1}\left(j_{n+1}\right) \cup(D-C)$ $\cup\left(D^{\prime}-C\right) \cup S(v)$. Note that this tube does not meet $S-S(v)$ and be sure that no two such tubes intersect. Add this tube to $T_{U}^{-1}\left(j_{n+1}\right)$. Make the above alterations for each vertex $v$ of $J_{n+1}$ and each $h \in t(H)$. Observe that we no longer have $P_{U}^{*}=\operatorname{cl}(M-U)$.

(5.9) We will now make the necessary alterations so that if $j_{n+1} \cap j_{n+1}^{\prime} \neq$ $\varnothing$, then each component of $T_{U}^{-1}\left(j_{n+1}\right)$ will meet $T_{U}^{-1}\left(j_{n+1}^{\prime}\right)$. Let us assume that the $S(v)^{\prime}$ 's were general positioned in (5.4) so that for each pair $h, h^{\prime} \in t(H)$ with $h \cap h^{\prime}$ an $m-1$ simplex, there are components $C_{h}$ and $C_{h^{\prime}}$ of $h-U$ and $h^{\prime}-U$ such that $C_{h} \cap C_{h^{\prime}}$ contains an $m-1$ cell and such that for each vertex $v^{\prime}$ of $J_{n+1}$ the closure of the component $D_{h}$ (resp., $D_{h^{\prime}}$ ) of $h-S\left(v^{\prime}\right)$ (resp., $h^{\prime}-S\left(v^{\prime}\right)$ ) containing $C_{h}$ (resp., $C_{h^{\prime}}$ ) does not intersect bd( $\left.h\right)-h \cap h^{\prime}$ (resp., 
$\left.\mathrm{bd}\left(h^{\prime}\right)-h \cap h^{\prime}\right)$. Choosing such a "general positioning" is not difficult (in fact, almost any "natural" choice will do).

For each $j_{n} \in J_{n}$, let $\tilde{t}\left(H \mid j_{n}\right)=\left\{h \in t\left(H \mid j_{n}\right) \mid h \nsubseteq A_{j_{n}}^{*}\right\}$. For each $h \in$ $\tilde{t}\left(H \mid j_{n}\right)$, let $r_{h}$ be the unique integer with

$$
h \in \mathrm{ST}^{L_{n+1}-r_{h}}\left(A_{j_{n}}^{*}, t\left(H \mid j_{n}\right)\right)-\mathrm{ST}^{L_{n+1}-r_{h}-1}\left(A_{j_{n}}^{*}, t\left(H \mid j_{n}\right)\right) \text {. }
$$

It follows from the choices of $Q^{\prime}, Q$ and $L_{n+1}$ in (5.2) that $r_{h} \geqslant 3 m^{2}+1$.

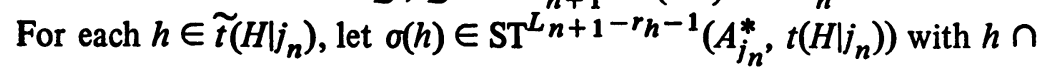
$\sigma(h)$ an $m-1$ simplex and let $C_{h}$ and $C_{\sigma(h)}$ be the components of $h-U$ and $\sigma(h)-U$ discussed above.

For each $a \in A_{j_{n}}$, define a collection $\Sigma(a)$ as follows. For each $j_{n}^{\prime} \in$ st $\left(j_{n}, J_{n}\right)$ with $j_{n}^{\prime} \neq j_{n}$, let $h_{j_{n}^{\prime}}^{\prime}, h_{j_{n}^{\prime}} \in t(H)$ with $h_{j_{n}^{\prime}}^{\prime} \subseteq a, h_{j_{n}^{\prime}} \subseteq R_{n}\left(j_{n}^{\prime}\right)$, and $\boldsymbol{h}_{j_{n}^{\prime}}^{\prime} \cap h_{j_{n}^{\prime}}$ an $m-1$ simplex; let $C_{h_{j_{n}^{\prime}}}$ and $C_{h_{j_{n}^{\prime}}}$ be components of $h_{j_{n}^{\prime}}^{\prime}-U$ and $h_{j_{n}^{\prime}}-U$ discussed above. Let

$$
\Sigma(a)=\left\{\left(C_{h_{j_{n}^{\prime}}^{\prime}}, C_{h_{j_{n}^{\prime}}}\right) \mid j_{n}^{\prime} \in \operatorname{st}\left(j_{n^{\prime}}, J_{n}\right) \text { and } j_{n}^{\prime} \neq j_{n}\right\}
$$

we emphasize that $\Sigma(a)$ contains exactly one pair $\left(C_{h_{j_{n}^{\prime}}^{\prime}}, C_{h_{j_{n}^{\prime}}}\right)$ for each $j_{n}^{\prime} \in$ $\operatorname{st}\left(j_{n}, J_{n}\right)$ with $j_{n}^{\prime} \neq j_{n}$.

Let

$$
\begin{aligned}
& \Gamma_{j_{n}}=\left\{\left(h, j_{n+1}\right) \mid h \in \tilde{t}\left(H \mid j_{n}\right) \text { and } j_{n+1} \in \mathrm{st}^{r^{+1}}\left(j_{n}, J_{n+1}\right)-\mathrm{st}^{r}{ }^{h}\left(j_{n}, J_{n+1}\right)\right\}, \\
& \Omega_{j_{n}}=\left\{\left(a, j_{n+1}\right) \mid a \in A_{j_{n}} \text { and } j_{n+1} \in \mathrm{st}^{L}{ }^{L+1}{ }^{+1}\left(j_{n}, J_{n+1}\right)-\mathrm{st}^{L}{ }^{L+1}\left(j_{n}, J_{n+1}\right)\right\} .
\end{aligned}
$$

For each $\left(h, j_{n+1}\right) \in \Gamma_{j_{n}}$ (resp., $\left.\left(a, j_{n+1}\right) \in \Omega_{j_{n}}\right)$ we will make $T_{U}^{-1}\left(j_{n+1}\right) \cap$ $\operatorname{int}(h)$ (resp., $\left.T_{U}^{-1}\left(j_{n+1}\right) \cap \operatorname{int}(a)\right)$ connected and then we will connect $T_{U}^{-1}\left(j_{n+1}\right) \cap C_{h}$ to $T_{U}^{-1}\left(j_{n+1}\right) \cap C_{\sigma(h)}$ (resp., $T_{U}^{-1}\left(j_{n+1}\right) \cap C_{h_{j}^{\prime}}$ to $T_{U}^{-1}\left(j_{n+1}\right) \cap C_{h_{j_{n}^{\prime}}}$ where $j_{n+1} \subseteq j_{n}^{\prime}$ and $\left.\left(C_{h_{j_{n}^{\prime}}^{\prime}}, C_{h_{j_{n}^{\prime}}}\right) \in \Sigma(a)\right)$. We emphasize that the same $\sigma(h)$ (resp., $\Sigma(a))$ is used for each pair $\left(h, j_{n+1}\right)$ (resp., $\left.\left(a, j_{n+1}\right)\right)$. The problem we need to remedy is that for $\left(h, j_{n+1}\right) \in \Gamma_{j_{n}}$ (resp., $\left(a, j_{n+1}\right) \in$ $\left.\Omega_{j_{n}}\right)$, if $j_{n+1}^{\prime} \in \mathrm{st}^{r_{h}+2}\left(j_{n}, J_{n+1}\right)-\mathrm{st}^{r+1}\left(j_{n}, J_{n+1}\right)\left(\right.$ resp., $j_{n+1}^{\prime} \in$ st $^{L_{n+1}+2}\left(j_{n}, J_{n+1}\right)-$ st $\left.^{L_{n+1}+1}\left(j_{n}, J_{n+1}\right)\right)$ with $j_{n+1}^{\prime} \cap j_{n+1} \neq \varnothing$, then $T_{U}^{-1}\left(j_{n+1}^{\prime}\right) \cap h=\varnothing$ (resp., $\left.T_{U}^{-1}\left(j_{n+1}^{\prime}\right) \cap a=\varnothing\right)$; hence, the components of $T_{U}^{-1}\left(j_{n+1}\right)$ in $h$ (resp., a) do not meet $T_{U}^{-1}\left(j_{n+1}\right)$. However, since $T_{U}^{-1}\left(j_{n+1}^{\prime}\right)$ $\cap \sigma(h) \neq \varnothing$ (resp., $T_{U}^{-1}\left(j_{n+1}^{\prime}\right) \cap h_{j_{n}^{\prime}} \neq \varnothing$ where $j_{n+1}^{\prime} \subseteq j_{n}^{\prime}$ ), once we have made the changes indicated above, we will have eliminated this problem. The changes we now describe are to be made for each $j_{n} \in J_{n}$.

For each $\left(h, j_{n+1}\right) \in \Gamma_{j_{n}}$ make the following alterations. Let $D$ and $D^{\prime}$ be components of $h-S$ with $\operatorname{cl}(D) \cap \operatorname{cl}\left(D^{\prime}\right)$ containing an $m-1$ cell; let $v$ be such that $\operatorname{cl}(D) \cap \operatorname{cl}\left(D^{\prime}\right)$ contains an $m-1$ cell in $S(v)$. Let $j_{n+1}^{1}, \ldots, j_{n+1}^{t}=$ 
$j_{n+1}$ be a chain (i.e., $j_{n+1}^{i} \cap j_{n+1}^{i+1} \neq \varnothing, i=1, \ldots, t-1$ ) in $\mathrm{st}^{{ }^{2}{ }^{+}+1}\left(j_{n}, J_{n+1}\right)$ with $j_{n+1}^{1} \subseteq j_{n}$, with $v \notin j_{n+1}^{i}$ for $i=t-2, \ldots, 1$, and with $j_{n+1}^{1} \cap N_{n}(v)=$ $\varnothing$. In view of the changes made in (5.8), $T_{U}^{-1}\left(j_{n+1}^{1}\right) \cap\left(\operatorname{int}\left(D \cup D^{\prime} \cup S(v)\right)\right)$ is connected; connect $T_{U}^{-1}\left(j_{n+1}^{2}\right) \cap D$ to $T_{U}^{-1}\left(j_{n+1}^{2}\right) \cap D^{\prime}$ with a tube in $\operatorname{int}\left(T_{U}^{-1}\left(j_{n+1}^{2}\right) \cup T_{U}^{-1}\left(j_{n+1}^{1}\right)\right) \cap\left(D \cup D^{\prime} \cup S(v)\right)$. The "new" $T_{U}^{-1}\left(j_{n+1}^{2}\right)$ meets $\operatorname{int}\left(D \cup D^{\prime} \cup S(v)\right)$ in a connected set; hence, we can connect $T_{U}^{-1}\left(j_{n+1}^{3}\right) \cap D$ to $T_{U}^{-1}\left(j_{n+1}^{3}\right) \cap D^{\prime}$ with a tube in $\operatorname{int}\left(T_{U}^{-1}\left(j_{n+1}^{3}\right) \cup T_{U}^{-1}\left(j_{n+1}^{2}\right)\right) \cap\left(D \cup D^{\prime} \cup\right.$ $S(v)$ ). In this manner, successively for $i=4, \ldots, t$, connect $T_{U}^{-1}\left(j_{n+1}^{i}\right) \cap D$ to $T_{U}^{-1}\left(j_{n+1}^{i}\right) \cap D^{\prime}$ with a tube $\operatorname{in} \operatorname{int}\left(T_{U}^{-1}\left(j_{n+1}^{i}\right) \cup T_{U}^{-1}\left(j_{n+1}^{i-1}\right)\right) \cap\left(D \cup D^{\prime}\right.$ $\cup S(v)$ ). Make the above alteration for each pair $D, D^{\prime}$ of components of $h-$ $S$ with $\operatorname{cl}(D) \cap \operatorname{cl}\left(D^{\prime}\right)$ containing an $m-1$ cell. Let $j_{n+1}^{1}, \ldots, j_{n+1}^{t}=j_{n+1}$ be a chain in $\operatorname{st}^{r_{h}+1}\left(j_{n}, J_{n+1}\right)$ with $j_{n+1}^{1} \subseteq j_{n}$. In view of (5.5.4), $T_{U}^{-1}\left(j_{n+1}^{1}\right)$ $\cap \operatorname{int}\left(C_{h} \cup C_{\sigma(h)}\right)$ is connected. As above, successively for $i=2, \ldots, t$, connect $T_{U}^{-1}\left(j_{n+1}^{i}\right) \cap C_{h}$ to $T_{U}^{-1}\left(j_{n+1}^{i}\right) \cap C_{\sigma(h)}$ with a tube in

$$
\operatorname{int}\left(T_{U}^{-1}\left(j_{n+1}^{i}\right) \cup T_{U}^{-1}\left(j_{n+1}^{i-1}\right)\right) \cap \operatorname{int}\left(C_{h} \cup C_{\sigma(h)}\right) \text {. }
$$

For each $\left(a, j_{n+1}\right) \in \Omega_{j_{n}}$ make the following alterations. Using the method in the preceding paragraph, make $T_{U}^{-1}\left(j_{n+1}\right) \cap$ int $(a)$ connected by connecting $T_{U}^{-1}\left(j_{n+1}\right) \cap D$ to $T_{U}^{-1}\left(j_{n+1}\right) \cap D^{\prime}$ for each pair of components $D, D^{\prime}$ of $a-\left(S \cup H^{m-1}\right)$ with $\operatorname{cl}(D) \cap \operatorname{cl}\left(D^{\prime}\right)$ containing an $m-1$ cell. (If connecting across an $m-1$ cell contained in $H^{m-1}$, then any chain $j_{n+1}^{1}, \ldots, j_{n+1}^{t}=$ $j_{n+1}$ in st ${ }^{L_{n+1}+1}\left(j_{n}, J_{n+1}\right)$ can be used; however, if the $m-1$ cell is contained in $S(v)$, then choose the chain so that $v \notin j_{n+1}^{i}$ for $i=t-2, \ldots, 1$ and so that $j_{n+1}^{1} \cap N_{n}(v)=\varnothing$.) Finally, let $j_{n}^{\prime}$ be such that $j_{n+1} \subseteq j_{n}^{\prime}$ and let $j_{n+1}^{1}, \ldots, j_{n+1}^{t}=j_{n+1}$ be a chain in $\operatorname{st}^{L_{n+1}+1}\left(j_{n}, J_{n+1}\right)$ with $j_{n+1}^{1} \subseteq j_{n}$ and $j_{n}^{i} \subseteq j_{n}^{\prime}$ for $i=2, \ldots, t$. In view of (5.5.4)

$$
\operatorname{int}\left(T_{U}^{-1}\left(j_{n+1}^{2}\right) \cup T_{U}^{-1}\left(j_{n+1}^{1}\right)\right) \cap \operatorname{int}\left(C_{h_{j_{n}^{\prime}}} \cup C_{h_{j_{n}^{\prime}}}\right)
$$

is connected; hence, we can connect $T_{U}^{-1}\left(j_{n+1}^{2}\right) \cap C_{h_{j_{n}^{\prime}}^{\prime}}$ to $T_{U}^{-1}\left(j_{n+1}^{2}\right) \cap C_{h_{j_{n}^{\prime}}}$ with a tube in this set. Now, successively for $i=3, \ldots, t$, connect $T_{U}^{-1}\left(j_{n+1}^{i}\right) \cap C_{h_{j_{n}^{\prime}}^{\prime}}$ to $T_{U}^{-1}\left(j_{n+1}^{i}\right) \cap C_{h_{j_{n}^{\prime}}}$ with a tube in

$$
\operatorname{int}\left(T_{U}^{-1}\left(j_{n+1}^{i}\right) \cup T_{U}^{-1}\left(j_{n+1}^{i-1}\right)\right) \cap\left(C_{h_{j_{n}^{\prime}}^{\prime}} \cup C_{h_{j_{n}^{\prime}}}\right) \text {. }
$$

(5.10) We now have that if $j_{n+1} \neq \varnothing$, then the intersection of each component of $T_{U}^{-1}\left(j_{n+1}\right)$ with $T_{U}^{-1}\left(j_{n+1}^{\prime}\right)$ contains an $m-1$ cell; and, in place of having $T_{U}^{-1}\left(j_{n+1}\right) \cap S(v)=\varnothing$ for each $v \in j_{n+1}$, we have that:

(5.10.1) For each vertex $v$ of $J_{n+1}$, each component of $\left\{T_{U}^{-1}\left(j_{n+1}\right)\right\}$ $\left.v \in j_{n+1}\right\}^{*}$ is a subset of $\operatorname{st}^{3}(h, t(H))^{*}$ or $\operatorname{st}^{3}(a, t(H))^{*}$ for some $h \in t(H)$ or $a \in \bigcup A_{j_{n}}$.

We will now outline a proof of the validity of (5.10.1). Let $B$ be a com- 
ponent of $\left\{T_{U}^{-1}\left(j_{n+1}\right) \mid v \in j_{n+1}\right\}^{*}$ and suppose that $B \cap S(v) \neq \varnothing$ (if $B \cap S(v)$ $=\varnothing$, then we are done). Let $s=\min \left\{s^{\prime}\right\}$ for some $j_{n} \in J_{n}$ and for some $h \in$ $\mathrm{ST}^{s^{\prime}}\left(A_{j_{n}}^{*}, t\left(H \mid j_{n}\right)\right)$ we have that $\left.B \cap S(v) \cap h \neq \varnothing\right\}$. Let us assume that $s \neq 0$ (the case $s=0$ is handled similarly; in particular, using the fact that $\mathrm{st}^{4}(a, t(H))^{*}$ $\cap \operatorname{st}^{4}\left(a^{\prime}, t(H)\right)^{*}=\varnothing, a \neq a^{\prime}$, one shows that if $B \cap S(v) \cap a \neq \varnothing$, then $B \subseteq$ $\left.\mathrm{st}^{3}(a, t(H))^{*}\right)$.

Let $j_{n} \in J_{n}$ be such that there is

$$
h \in \mathrm{ST}^{s}\left(A_{j_{n}}^{*}, t\left(H \mid j_{n}\right)\right)-\mathrm{ST}^{s-1}\left(A_{j_{n}}^{*}, t\left(H \mid j_{n}\right)\right)
$$

with $B \cap S(v) \cap h \neq \varnothing$ (it will turn out that both $j_{n}$ and $h$ are unique; this is not obvious). Recalling the definition of $r_{h}$, it certainly is the case that $s=L_{n+1}$ $-r_{h}$. One of the "controls" governing the changes made in $\$(5.9)$ involved limiting the possible intersection of $T_{U}^{-1}\left(j_{n+1}\right)$ with $S(v)$ for each $j_{n+1} \in$ $\operatorname{st}\left(v, J_{n+1}\right)$ (recall the carefully chosen chains used in the final two paragraphs of (5.9)); in particular, one can verify that we must have that $v \in \operatorname{st}^{q}\left(j_{n}, J_{n+1}\right)^{*}$ - st ${ }^{q-1}\left(j_{n}, J_{n+1}\right)^{*}$ where $q$ is equal to one of the following: $r_{h}+1, r_{h}$, or $r_{h}-1$. Let us assume that $q=r_{h}$ (the remaining two cases can be handled similarly). Let $h_{1}, \ldots, h_{t}$ be all the sets in $\mathrm{ST}^{L_{n+1}-r_{h}+1}\left(A_{j_{n}}^{*}, t\left(H \mid j_{n}\right)\right)-$ $\mathrm{ST}^{L_{n+1}-r_{h}}\left(A_{j_{n}}^{*}, t\left(H \mid j_{n}\right)\right)$ for which $\sigma\left(h_{i}\right)=h, i=1, \ldots, t$. Letting $G=h \cup$ $\sigma(h) \cup\left(\cup_{i=1}^{t} h_{i}\right)$, we claim that $B \subseteq \operatorname{int}(G)$; this fact can be deduced as follows. Let $j_{n+1} \in \operatorname{st}\left(v, J_{n+1}\right)$; since $v \in \mathrm{st}^{r} h\left(j_{n}, J_{n+1}\right)^{*}-\mathrm{st}^{r}{ }^{-1}\left(j_{n}, J_{n+1}\right)^{*}$, we must have that

$$
\begin{aligned}
& j_{n+1} \in \mathrm{st}^{r}{ }^{h}\left(j_{n}, J_{n+1}\right)-\mathrm{st}^{r}{ }^{-1}\left(j_{n}, J_{n+1}\right) \text { or } \\
& j_{n+1} \in \mathrm{st}^{r}{ }^{+1}\left(j_{n}, J_{n+1}\right)-\mathrm{st}^{r}\left(j_{n}, J_{n+1}\right) .
\end{aligned}
$$

We need to know where $T_{U}^{-1}\left(j_{n+1}\right)$ intersects bd(G). (Before the changes in (5.9) were made, we had that $T_{U}^{-1}\left(j_{n+1}\right) \cap H^{m-1} \subseteq R_{n}\left(j_{n}^{\prime}\right)$ where $j_{n+1} \subseteq j_{n}^{\prime}$; since $G \cap R_{n}\left(j_{n}^{\prime}\right)=\varnothing, T_{U}^{-1}\left(j_{n+1}\right) \cap \mathrm{bd}(G)$ is completely determined by the changes made in (5.9).) Letting $C_{1}=\left\{C_{h^{\prime}} \cap C_{\sigma(h)} \mid h^{\prime} \in \mathrm{ST}^{s}\left(A_{j_{n}}^{*}, t\left(H \mid j_{n}\right)\right)\right.$ with $h^{\prime} \neq h$ and $\left.\sigma\left(h^{\prime}\right)=\sigma(h)\right\}^{*}$ and $C_{2}=\left\{C_{h^{\prime}} \cap C_{h_{i}} \mid h^{\prime} \in \mathrm{ST}^{s+2}\left(A_{j_{n^{\prime}}^{*}}^{*}, t\left(H \mid j_{n}\right)\right)\right.$ with $\sigma\left(h^{\prime}\right)=h_{i}$ for some $\left.i=1, \ldots, t\right\}^{*}$, certainly, we have that $T_{U}^{-1}\left(j_{n+1}\right) \cap \operatorname{bd}(G)$ $\subseteq C_{1} \cup C_{2}$. However, the choice of minimal $s$ implies that $B \cap S(v) \cap \sigma(h)=\varnothing$; therefore, because of the choice of component $C_{\sigma(h)}$ in (5.9), we have that $B \cap$ $C_{1}=\varnothing$. In addition, if $\sigma\left(h^{\prime}\right)=h_{i}$ for some $i=1, \ldots, t$, then $h^{\prime} \in$ $\mathrm{ST}^{s+2}\left(A_{j_{n}}^{*}, t\left(H \mid j_{n}\right)\right)-\mathrm{ST}^{s+1}\left(A_{j_{n}}^{*}, t\left(H \mid j_{n}\right)\right)$ and $T_{U}^{-1}\left(j_{n+1}\right) \cap h^{\prime}=\varnothing$; therefore $B \cap C_{2}=\varnothing$. Hence, we have that $B \subseteq \operatorname{int}(G)$.

In dealing with the case that $q=r_{h}-1$, in place of the set $G$, it is necessary to use the set 


$$
\begin{aligned}
G^{\prime}= & \left(h \cup \sigma(h) \cup\left(\bigcup_{i=1}^{t} h_{i}\right)\right) \\
& \cup\left\{h^{\prime} \mid h^{\prime} \in \mathrm{ST}^{s+2}\left(A_{j_{n}}^{*}, t\left(H \mid j_{n}\right)\right) \text { with } \sigma\left(h^{\prime}\right)=h_{i} \text { for some } i=1, \ldots, t\right\}^{*} .
\end{aligned}
$$

If $q=r_{h}+1$, then the set $G$ suffices.

(5.11) We are now in a position to construct a collection of $m$ cells $A_{j_{n+1}}^{\prime}$ ', for each $j_{n+1} \in J_{n+1}$, satisfying condition (5.1.7) ${ }^{n+1}$ for the triple $T_{U}: P_{U} \rightarrow$ $J_{n+1}$. For each $j_{n+1} \in J_{n+1}$ and for each component $W$ of $T_{U}^{-1}\left(j_{n+1}\right)$ do the following. Let $h \in t(H)$ with $W \cap T_{U}^{-1}\left(j_{n+1}^{\prime}\right) \cap$ int $(h)$ containing an $m-1$ cell for each $j_{n+1}^{\prime} \in \operatorname{st}\left(j_{n+1}, J_{n+1}\right)$; for certain $W$ such an $h$ existed before the changes in (5.9) were made and for the others the changes in (5.9) yield such an $h$. Choose an $m$ cell in int $(W) \cap \operatorname{int}(h)$ of diameter less than $4 / 2^{n+1}$ and, for each $j_{n+1}^{\prime} \in \operatorname{st}\left(j_{n+1}, J_{n+1}\right)$, connect $T_{U}^{-1}\left(j_{n+1}^{\prime}\right) \cap h$ to this $m$ cell with a tube in $\operatorname{int}\left(W \cup T_{U}^{-1}\left(j_{n+1}^{\prime}\right)\right) \cap \operatorname{int}(h)$; this $m$ cell is to be an element of $A_{j_{n+1}}^{\prime}$.

(5.12) Let $V=\operatorname{cl}\left(U-\left\{T_{U}^{-1}\left(j_{n+1}\right) \mid j_{n+1} \in J_{n+1}\right\}^{*}\right)$ and let $B_{2}$ be a subdivision of $B_{1}$ which subdivides $V$. In this section we will construct a (particular) p.l. mapping from $V$ to $N$ and in (5.13) we will use this mapping to "enlarge" the $T_{U}^{-1}\left(j_{n+1}\right)$ 's so that they "fill" all of $M$.

Using the machinery set up in (5.2) and Proposition A from the appendix, we now construct a pl. mapping $g: V \rightarrow N$ satisfying:

(5.12.1) $g(S(v) \cap V) \cap \operatorname{st}\left(v, J_{n+1}\right)^{*}=\varnothing$ for each vertex $v$ of $J_{n+1}$.

(5.12.2) If $\sigma \in B_{2}$ and $\sigma \subseteq$ bd(V), then $g(\sigma) \subseteq \bigcap\left\{j_{n+1} \mid \sigma \subseteq T_{U}^{-1}\left(j_{n+1}\right)\right\}$.

(5.12.3) $g\left(V \cap R_{n}\left(j_{n}\right)\right) \subseteq \mathrm{st}^{3 m^{2}}\left(j_{n}, \beta^{l} L\right)^{*}$ for each $j_{n} \in J_{n}$.

For each vertex $v \in J_{n+1}$, let $w_{v}$ be a vertex of $\beta^{l^{\prime}-3} L$ with $v \in$ $\operatorname{st}\left(w_{v}, \beta^{l^{\prime}-2} L\right)^{*}$ and let $D_{v}=\operatorname{st}\left(\operatorname{st}\left(w_{v}, \beta^{l^{\prime}-2} L\right)^{*}, \beta^{l^{\prime}} L\right)^{*} ;$ note that $\operatorname{st}\left(v, J_{n+1}\right)^{*} \subseteq$ $\operatorname{int}\left(D_{v}\right)$; see Figure 1. Define $g$ on $\operatorname{bd}(V)$ inductively, for $i=0, \ldots, m-1$, by mapping each $i$ simplex $\sigma \in B_{2}$ into $\bigcap\left\{j_{n+1} \mid \sigma \subseteq T_{U}^{-1}\left(j_{n+1}\right)\right\}$ (see (1.1) for more details). Observe that the set $S \cap \operatorname{bd}(V)$ is completely determined by the changes made in (5.8); in particular, if $\sigma \subseteq S \cap$ bd( $V)$, then there is a unique vertex $v$ of $J_{n+1}$ with $\sigma \subseteq S(v) \cap \operatorname{bd}(V)$. Furthermore, we have that $g(\sigma) \cap \operatorname{int}\left(D_{v}\right)=\varnothing$ (since the diameter of $D_{v}$ is less than $\eta, D_{v} \subseteq N_{\eta}(v)$; now recall the condition in the fourth sentence of $\S(5.8)$ ).

If $y$ is a vertex of $B_{2}$ in $S \cap V-\operatorname{bd}(V)$, then let $v_{1}, \ldots, v_{s}$ be all the vertices of $J_{n+1}$ with $y \in S\left(v_{i}\right), i=1, \ldots, s$; because of the general positioning done in (5.4), we have that $s \leqslant m$. Using the machinery set up in (5.2), we can find an integer $r, 0<r \leqslant 3 m$, such that $D_{v_{i}} \cap \operatorname{bd}\left(\operatorname{st}^{r}\left(\bigcap\left\{j_{n} \mid y \in R_{n}\left(j_{n}\right)\right\}, \beta^{l} L\right)^{*}\right)=$ $\varnothing$ for each $i=1, \ldots, s$; in general, the $D_{v_{i}}$ 's will be located throughout $M$; we are concerned with avoiding those $D_{v_{i}}$ 's contained in 


$$
\mathrm{st}^{3 m}\left(\bigcap\left\{j_{n} \mid y \in R_{n}\left(j_{n}\right)\right\}, \beta^{l} L\right)^{*} \text {. }
$$

Let $g(y) \in \operatorname{int}\left(\operatorname{st}^{r}\left(\bigcap\left\{j_{n} \mid y \in R_{n}\left(j_{n}\right)\right\}_{3} \beta^{l} L\right)^{*}\right)$ but $g(y) \notin \operatorname{int}\left(\bigcup_{i=1}^{s} D_{v_{i}}\right)$.

If $\tau$ is a 1 simplex of $B_{2}$ in $S \cap V(\tau \nsubseteq \mathrm{bd}(V))$, then let $v_{1}, \ldots, v_{s}$ be all the vertices of $J_{n+1}$ with $\tau \subseteq S\left(v_{i}\right), i=1, \ldots, s$ (we have that $s \leqslant m-1$ ). Let $r$ be an integer, $3 m<r \leqslant 2(3 m)$, with

$$
D_{v_{i}} \cap \operatorname{bd}\left(\mathrm{st}^{r}\left(\cap\left\{j_{n} \mid \tau \subseteq R_{n}\left(j_{n}\right)\right\}, \beta^{l} L\right)^{*}\right)=\varnothing
$$

for each $i=1, \ldots, s$. Extend $g$ to $\tau$ by mapping $\tau$ into

$$
\left.\operatorname{int}\left(\operatorname{st}^{r}\left(\bigcap j_{n} \mid \tau \subseteq R_{n}\left(j_{n}\right)\right\}, \beta^{l} L\right)^{*}\right)
$$

but with $g(\tau) \cap \operatorname{int}\left(\bigcup_{i=1}^{s} D_{v_{i}}\right)=\varnothing$ by using Proposition A (see the Appendix) with $q=l^{\prime}$ and $\left.B=\operatorname{st}^{r}\left(\bigcap j_{n} \mid \tau \subseteq R_{n}\left(j_{n}\right)\right\}, \beta^{l} L\right)^{*}$ (ignore those $D_{v_{i}}$ 's not contained in $B)$.

In general, to extend $g$ from the $q$ skeleton of $S \cap V$ to the $q+1$ skeleton of $S \cap V$, let $\tau$ be a $q+1$ simplex in $S \cap V\left(\tau \underline{q}\right.$ bd(V)) and let $v_{1}, \ldots, v_{s}$ be all the vertices of $J_{n+1}$ with $\tau \subseteq S\left(v_{i}\right), i=1, \ldots, s$ (we have that $s \leqslant m-$ $q-1$ ). Let $r$ be an integer, $q(3 m)<r \leqslant(q+1) 3 m \ldots$ (continue reading from the third line of the preceding paragraph).

Extend $g$ to all of $V$ by mapping a $q$ simplex $\tau \subseteq V$ ( $\tau \subseteq S$ into $\left.\operatorname{int}\left(\mathrm{st}^{3 m^{2}}\left(\bigcap j_{n} \mid \tau \subseteq R_{n}\left(j_{n}\right)\right\}, \beta^{l} L\right)^{*}\right)$; more precisely, do this inductively beginning with the vertices of $B_{2}$ in $V-S$.

(5.13) Let $B_{3}$ be a subdivision of $B_{2}$ such that $g$ maps each simplex of $B_{3}$ contained in $V$ linearly into a simplex of $J_{n+1}$.

Let $\tau \in t\left(B_{3}\right)$ with $\tau \nsubseteq P_{U}^{*}$ and with $\tau \cap P_{U}^{*} \cap \operatorname{int}(h)$ containing an $m-1$ cell where $h \in t(H)$ is such that $\tau \subseteq h$. Let $D$ be the component of $h-S$ with $\tau \subseteq \operatorname{cl}(D)$. Let $j_{n+1} \in J_{n+1}$ be such that $\tau \cap T_{U}^{-1}\left(j_{n+1}\right) \cap$ int $(h)$ contains an $m-1$ cell; property (5.12.2) implies that if $j_{n+1}^{\prime} \in J_{n+1}$ is such that $g(\tau) \subseteq$ $j_{n+1}^{\prime}$, then $j_{n+1} \cap j_{n+1}^{\prime} \neq \varnothing$. Properties (5.12.3) and (5.6.1) and the comment in the last sentence of (5.3) guarantee that we can run a tube in

$$
\operatorname{int}\left(T_{U}^{-1}\left(j_{n+1}\right) \cup \tau\right) \cap D
$$

connecting $\tau$ to $T_{U}^{-1}\left(j_{n+1}^{\prime}\right)$; add $\tau$ and this tube to $T_{U}^{-1}\left(j_{n+1}^{\prime}\right)$ and replace $T_{U}^{-1}\left(j_{n+1}\right)$ by $\mathrm{cl}\left(T_{U}^{-1}\left(j_{n+1}\right)\right.$-tube). Let $T_{U, 1}: P_{U, 1} \rightarrow J_{n+1}$ denote this new triple.

Repeat the alterations of the preceding paragraph using $T_{U, 1}: P_{U, 1} \rightarrow$ $J_{n+1}$ in place of $T_{U}: P_{U} \rightarrow J_{n+1}$ subject to the following modifications. Choose $\tau \in t\left(B_{3}\right)$ so that $\tau \cap P_{U, 1}^{*} \cap$ int $(h)$ contains an $m-1$ cell not in $S$; and if $\tau \cap P_{U, 1}^{*} \cap \operatorname{int}(h)$ is not contained in $\operatorname{bd}(V)$, then read " $g$ being a continuous 
function" in place of "property (5.12.2)". Call the new triple $T_{U, 2}: P_{U, 2} \rightarrow$ $J_{n+1}$.

Continue repeating the above alterations using the "new" triple each time until, after say $q$ times, we have $P_{U, q}^{*}=M$.

(5.14) Finally, let $R_{n+1}^{\prime}=T_{U, q}^{-1}$ and $K_{n+1}^{\prime}=P_{U, q}$. We leave to the reader to verify that the triple $R_{n+1}^{\prime}: J_{n+1} \rightarrow K_{n+1}^{\prime}$ satisfies properties $(5.1 .1)^{n+1}$ $(5.1 .8)^{n+1}$ except that the collection of components of sets in $K_{n+1}^{\prime}$ may not be simple and that some components of $R_{n+1}^{\prime}\left(j_{n+1}\right)$ may contain more than one of the $m$ cells in $A_{j_{n+1}}^{\prime}$. Property (5.1.8) ${ }^{n+1}$ follows from properties (5.10.1) and (5.12.1) (it is also necessary to observe that, because of (5.12.3), if $C$ is a component of $\left\{T_{U}^{-1}\left(j_{n+1}\right) \mid v \in j_{n+1}\right\}$ with $C \cap S(v) \neq \varnothing$, then $C$ is not changed by the alterations made in (5.13)). Let $B_{4}$ be a subdivision of $B_{3}$ such that each element of $K_{n+1}^{\prime}$ is a union of sets in $t\left(B_{4}\right)$. By running "small" tubes in various of the sets $\operatorname{int}\left(\operatorname{st}\left(v, \beta^{2} B_{4}\right)\right)$ where $v$ is a vertex of $\beta^{1} B_{4}$, the collection $K_{n+1}^{\prime}$ can be altered so that the collection of components of sets in $K_{n+1}^{\prime}$ is simple (the tubes should be small enough so that conditions (5.1.5) $)^{n+1}$ and (5.1.6) $)^{n+1}$ still hold); at last we have our triple $R_{n+1}: J_{n+1} \rightarrow K_{n+1}$. Let $A_{j_{n+1}}$ be a subset of $A_{j_{n+1}}^{\prime}$ chosen so that each component of $R_{n+1}\left(j_{n+1}\right)$ contains exactly one $m$ cell. This completes the proof of (5.1).

(5.15) The following corollary follows from Theorem (5.0) in much the same way as Corollary (3.7.1) followed from Theorem (3.0).

(5.15.1) COROLlaRY. Let $M^{m}, N^{n}$ be compact connected p.l. manifolds with $n \geqslant m \geqslant 3$, let $f$ be an open mapping of $M$ onto $N$, and let $\epsilon>0$. Then there is a light open mapping $g$ from $M$ onto $N$ with $d(f(x), g(x))<\epsilon$ for each $x \in M$ and with each $g^{-1}(y)$ homeomorphic to a Cantor set.

OUtLine OF Proof. Mimic the proof of Corollary (3.7.1) in order to find a triple $T: P \rightarrow t(L)$ satisfying the conditions of Theorem (5.0); in place of the statement " $U_{\sigma}=f^{-1}\left(\operatorname{int}\left(\operatorname{st}^{2}(\sigma, t(L)) *\right)\right)$ as an open connected set" use the fact that "each component of $U_{\sigma}$ is open and maps by $f$ onto int $\left.\left(\mathrm{st}^{2}(\sigma, t(L))\right)^{*}\right)$."

The following results are immediate consequences of (3.7.1), (5.15.1), and (4.0).

(5.15.2) CoROllary. Let $M^{m}$ and $N^{n}$ be compact, connected p.l. manifolds with $n \geqslant m \geqslant 3$. If $f$ is a monotone mapping of $M$ onto $N$, then $f$ can be uniformly approximated by light open mappings.

(5.15.3) Corollary. Let $M^{m}$ and $N^{n}$ be as above. A mapping $f$ from $M$ to $N$ is homotopic to a light open mapping of $M$ onto $N$ if and only if the index of $f_{*}\left(\pi_{1}(M)\right)$ in $\pi_{1}(N)$ is finite. 
APPENDIX. The following somewhat peculiar result is needed in the proof of (5.1); in fact, it is the "key step" in extending the technique used in [24] to construct open mappings to a technique to construct light open mappings. The reader is referred to [6] for the results from pl. topology used in the proof.

(A) Proposition. Let $M^{m}$ be a compact, connected p.l. manifold with triangulation $L$, let $B$ be a subcomplex of $L$ p.l. homeomorphic to an $n$ ball, and let $q$ be an integer larger than 4. Let $v_{1}, \ldots, v_{s}$ be vertices of $\beta^{q-3} L$ with each set $\operatorname{st}\left(v_{i}, \beta^{q-2} L\right)^{*} a$ subset of $\operatorname{int}(B), i=1, \ldots$, s. Then

$$
\operatorname{int}(B)-\operatorname{int}\left(\bigcup_{i=1}^{s} \operatorname{st}\left(\operatorname{st}\left(v_{i}, \beta^{q-2} L\right)^{*}, \beta^{q} L\right)^{*}\right)
$$

is $m-s-1$ connected.

Proof. Observe that there is no constraint on how $B$ meets $\partial M$ and recall that $\operatorname{int}(B)$ is the topological interior. Let $K=\left\{\left\langle v_{i_{1}}, \ldots, v_{i_{r}}\right\rangle \in \beta^{q-3} L \mid\right.$ $\left\{v_{i_{1}}, \ldots, v_{i_{r}}\right\} \subseteq\left\{v_{1}, \ldots, v_{s}\right\}$ and $\left.\bigcap_{j=1}^{r} \operatorname{st}\left(v_{i_{j}}, \beta^{q-2} L\right)^{*} \neq \varnothing\right\}$. Then $K$ is a full subcomplex of $\beta^{q-3} L$ and $\operatorname{int}(B)-\operatorname{int}\left(\bigcup_{i=1}^{s} \operatorname{st}\left(v_{i}, \beta^{q-2} L\right)^{*}\right)$ is a strong deformation retract of $\operatorname{int}(B)-K$. Since $\bigcup_{i=1}^{r} \operatorname{st}\left(\operatorname{st}\left(v_{i}, \beta^{q-2} L\right)^{*}, \beta^{q} L\right)^{*}$ is a regular neighborhood of $\bigcup_{i=1}^{s} \operatorname{st}\left(v_{i}, \beta^{q-2} L\right)^{*}$, the

$$
\operatorname{int}(B)-\operatorname{int}\left(\bigcup_{i=1}^{s} \operatorname{st}\left(\operatorname{st}\left(v_{i}, \beta^{q-2} L\right)^{*}, \beta^{q} L\right)^{*}\right)
$$

is a strong deformation retract of $\operatorname{int}(B)-K$. Finally, observe that the dimension of $K$ is at most $s-1$ so that the result follows by general positioning.

\section{REFERENCES}

1. R. D. Anderson, Open mappings of compact continua, Proc. Nat. Acad. Sci. U.S.A. 42 (1956), 347-349. MR 17, 1230.

2. S. Armentrout, Concerning cellular decompositions of 3-manifolds that yield 3-manifolds, Trans. Amer. Math. Soc. 133 (1968), 307-332. MR 37 \#5859.

3. S. Eilenberg, On the problems of topology, Ann. of Math. (2) 50 (1949), 247260. MR 10, 726.

4. E. E. Floyd and F. B. Jones, Gordon T. Whyburn 1904-1969, Bull. Amer. Math. Soc. 77 (1971), 57-72. MR 42 \#1639.

5. J. G. Hocking, Approximations to monotone mappings on non-compact two-dimensional manifolds, Duke Math. J. 21 (1954), 639-651. MR 16, 158.

6. J. F. P. Hudson, Piecewise linear topology, Univ. of Chicago Lecture Notes, Benjamin, New York and Amsterdam, 1969. MR 40 \#2094.

7. L. V. Keldyš, Monotone mappings of a cube onto a cube of higher dimension, Mat. Sb. 41(83) (1957), 129-158. (Russian) MR 19, 874.

8. - Transformation of a monotone irreducible mapping into a monotone-interior mapping and a monotone-interior mapping of the cube onto the cube of higher dimension, Dokl. Akad. Nauk. SSSR 114 (1957), 472-475. (Russian) MR 19, 972.

9. - Example of a monotone irreducible mapping of a three-dimensional cube onto a four-dimensional one, Dokl. Akad. Nauk. SSSR 103 (1955) 957-960. (Russian) MR 17, 992.

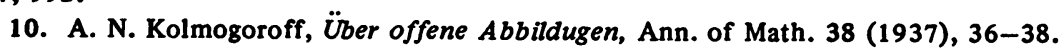


11. L. F. McAuley, Some fundamental theorems and problems related to monotone mappings, Proc. First Conf. on Monotone Mappings and Open Mappings (SUNY at Binghamton, Binghamton, N. Y., 1970), State Univ. of New York at Binghamton, N. Y., 1971, pp. 1-36. MR 44 \#4722.

12. - Open mappings and open problems, Topology Conference (Arizona State Univ., Tempe, Ariz., 1967), Arizona State Univ., Tempe, Ariz., 1968, pp. 184-202. MR 39 \#2134.

13. R. L. Moore, Concerning upper semi-continuous collections of continua, Trans. Amer. Math. Soc. 27 (1925), 416-428.

14. J. H. Roberts and N. E. Steenrod, Monotone transformations of 2-dimensional manifolds, Ann. of Math. (2) 39 (1938), 851-862.

15. S. Smale, A Vietoris mapping theorem for homotopy, Proc. Amer. Math. Soc. 8 (1957), 604-610. MR 19, 302.

16. S. Stoïlow, Sur les transformations continues et la topologie des fonctions analytiques, Ann. Sci. École Norm. Sup. (3) 45 (1928), 347-382.

17. L. C. Siebenmann, Approximating cellular maps by homeomorphisms, Topology 11 (1972), $271-294$. MR 45 \#4431.

18. D. C. Wilson, Open mappings of the universal curve onto continuous curves, Trans. Amer. Math. Soc. 168 (1972), 497-515. MR 45 \#7682.

19. Open mappings on manifolds and a counterexample to the Whyburn conjecture, Duke Math. J. 40 (1973), 705-716. MR 47 \#9522.

20. Monotone mappings of manifolds onto cells, Proc. First Conf. on Monotone Mappings and Open Mappings (SUNY at Binghamton, Binghamton, N. Y., 1970), State Univ. of New York at Binghamton, Binghamton, N. Y., 1971, pp. 37-54. MR 43 \#5501.

21. J. W. T. Youngs, Homeomorphic approximations to monotone mappings, Duke Math. J. 15 (1948), 87-94. MR 9, 524.

22. S. Smale, $A$ note on open maps, Proc. Amer. Math. Soc. 8 (1957), 391-393. MR 19, 158. \#1007.

23. E. H. Spanier, Algebraic topology, McGraw-Hill, New York, 1966. MR 35

24. J. Walsh, Monotone and open mappings on manifolds. I, Trans. Amer. Math. Soc. 209 (1975), 419-432.

\section{SCHOOL OF MATHEMATICS, INSTITUTE FOR ADVANCED STUDY, PRINCETON, NEW JERSEY 08540}

Current address: Department of Mathematics, University of Oklahoma, Norman, Oklahoma 73069 